\title{
La experiencia de alfabetización de personas adultas como proceso de aprendizaje en la formación docente
}

\section{The Experience of Adult Literacy as a Learning Process in the Formation of Future Educators}

\author{
Luz Emilia Flores Davis \\ División de Educación Básica del \\ Centro de Investigación y Docencia en Educación \\ Universidad Nacional \\ Heredia, Costa Rica \\ Ana María Hernández Segura ${ }^{2}$ \\ División de Educación Básica del \\ Centro de Investigación y Docencia en Educación (CIDE) \\ Universidad Nacional \\ Heredia, Costa Rica
}

Recibido 01 de febrero de $2010 \bullet$ Aceptado 17 de marzo de 2010

\begin{abstract}
Resumen. El aprendizaje se construye en la significación, por lo que promover experiencias que generen sentido y gozo, constituye un reto en la formación docente. En este artículo se comparten las reflexiones emanadas de una investigación que recaba la experiencia de alfabetización de personas adultas que realizan estudiantes universitarias o universitarios en el marco de su formación, la cual crea un proceso de aprendizaje que propicia el respeto a la diversidad y la valoración de la lectura y escritura como liberación del ser humano. El trabajo evidencia los aprendizajes construidos, las formas de respeto a la diversidad que emergen de la experiencia de alfabetización, y el valor que el estudiantado otorga a la lectura y escritura como expresión liberadora.
\end{abstract}

Palabras clave. Aprendizaje, formación docente, alfabetización de personas adultas.

Abstract. Learning is constructed when it is significant, therefore, promoting experiences that can make sense and joy, which constitutes a challenge in the formation of future educators. In this article, we share the considerations emanated from an investigation that gathers the experience of alphabetizing adults,

\footnotetext{
Doctora en Educación con mención en Mediación Pedagógica. Máster en Educación Superior. Catedrática universitaria. Ha ocupado los cargos de Directora de Docencia, Directora de Desarrollo Profesional y Cooperación Universitaria, Presidenta de la Junta de Becas y Coordinadora de la Comisión Técnica de Admisión de la Universidad Nacional. Actualmente, se desempeña como investigadora, extensionista y docente en la División de Educación Básica del Centro de Investigación y Docencia en Educación (CIDE) de la Universidad Nacional, Costa Rica y docente en la Maestría en Danza con mención en Formación Dancística del Centro de Investigación, Docencia y Extensión Artística (CIDEA), Universidad Nacional, Costa Rica. Correo electrónico: luzdeflores@yahoo.com

2 Máster en Educación con Mención en Docencia Universitaria, Licenciada en Educación Preescolar de la Universidad Nacional, Costa Rica y Terapista de Lenguaje, Madrid, España. Actualmente se desempeña como docente, investigadora y extensionista en la División de Educación Básica del Centro de Investigación y Docencia en Educación (CIDE) de la Universidad Nacional, Costa Rica, coordinó el equipo de Mejoramiento continuo de las carreras acreditadas de la División de Educación Básica (DEB) del Centro de Investigación y Docencia en Educación (CIDE) de la Universidad Nacional, Costa Rica, y fue integrante de la Comisión de Carrera Académica de la Universidad Nacional de Costa Rica.
} 
carried out by college students as part of their formation. As a result, this creates a learning process that favors the respect to diversity and the value of reading and writing as the liberation of mankind. The work displays the learnings constructed, the ways of respecting diversity that emerge from the alphabetizing experience, and the value that the students give to reading and writing as a liberating way of expression.

Key words. Learning, Formation of Educators, Literacy.

\section{Introducción}

La experiencia de alfabetización de personas adultas, facilitada por estudiantes universitarias o universitarios en el marco de la formación docente, constituye un proceso de aprendizaje que propicia el respeto a la diversidad y la valoración de la lectura y escritura como liberación del ser humano. Lo anterior se confirma mediante la indagación realizada para conocer los aprendizajes generados por los estudiantes y las estudiantes en un proceso de alfabetización de una persona adulta, experiencia que forma parte de sus cursos universitarios. Además, permite ubicar las variadas formas de respeto a la diversidad que emergen de esa experiencia de alfabetización. Todo ello para conocer el valor que el estudiantado otorga a la lectura y escritura como expresión liberadora.

En ese proceso participaron 68 estudiantes de primer año de la carrera de Pedagogía de la División de Educación Básica del CIDE, en la Universidad Nacional de Costa Rica. Uno de los grupos participantes corresponde al énfasis de I y II Ciclos y dos grupos pertenecen al énfasis de Educación Especial. Dichos estudiantes promovieron la alfabetización de setenta y cinco personas.

Para llevar a cabo la indagación se contó con un reporte de cada estudiante, en el cual comparte la descripción y el análisis de su experiencia, así como con un ensayo donde enfoca el significado del proceso vivido. Además, se elaboró un cuestionario con preguntas abiertas y se realizaron relatos inducidos, entrevistas a profundidad y se tomaron fotografías como evidencias del proceso.

\section{Contextualización de la experiencia}

La Universidad Nacional de Costa Rica declara en el Preámbulo de su Estatuto Orgánico que "La investigación, la docencia y la extensión y otras formas de producción académica, constituyen los pilares básicos de la actividad universitaria. Mediante la acción conjunta de estas áreas, la Universidad Nacional devela los problemas fundamentales de la sociedad, propone alternativas, y forma profesionales conscientes de las necesidades de la sociedad y del mundo en que vive" (Universidad Nacional, 1993, p. 3).

En el año de 1983 se crea el Centro de Investigación y Docencia en Educación, CIDE, a partir de la Escuela de Educación que formaba parte de la Facultad de Filosofía y Letras. Este Centro está formado por unidades académicas que integran su trabajo desde su objeto de estudio: la educación.

Una de las Unidades Académicas que conforman el CIDE es la División de Educación Básica (DEB), la cual ofrece las siguientes carreras: Pedagogía en I y II Ciclos, Pedagogía en Educación Preescolar, Pedagogía en Educación Especial, Pedagogía en I y II Ciclos en Inglés, Maestría en Pedagogía con énfasis en Atención a la Diversidad de los Procesos Educativos y Maestría en Pedagogía con énfasis en Desarrollo y Atención Integral a la Primera Infancia. De las carreras 
mencionadas, tres están reacreditadas ante el Sistema Nacional de Acreditación de la Educación Superior, (SINAES), lo que implica que cumplen con una serie de estándares de calidad en su oferta educativa.

A lo largo de la historia de la División de Educación Básica, se han desarrollado diversos enfoques epistemológicos, los cuales han intentado dar respuestas oportunas a grandes desafíos pedagógicos. Dichos enfoques han sido asimilados e interpretados desde la perspectiva de un análisis situacional del hecho educativo. En la actualidad, el reto de la consolidación de una praxis pedagógica crítica y creativa sigue vigente. La integración de las áreas académicas de la Universidad y la relación entre teoría y práctica en los procesos de formación han llevado a la realización de experiencias concretas como la que se presenta en este artículo, la cual corresponde al ámbito de la lectura y escritura.

Para concretar estas aspiraciones, la DEB cuenta con planes de estudio para cada una de las carreras mencionadas, las que a su vez comparten una estructura en común conformada por áreas disciplinarias, ejes curriculares, temas transversales, principios metodológicos, principios de evaluación, además de cursos del componente común pedagógico y oferta de cursos optativos.

En lo que respecta a las áreas disciplinarias, destacan la científica-pedagógica, de desarrollo humano integral y socio-histórica cultural. Por su parte, los temas transversales permiten que el estudiantado esté en contacto con la realidad social, estos son: multiculturalidad, género, derechos humanos, ecología y sostenibilidad, poblaciones diferenciadas, educación para la paz y marginalidad.

Los principios metodológicos que se proponen en los planes de estudio son los siguientes: enseñanza constructiva, funcionalidad de los aprendizajes, significatividad de los aprendizajes, interactividad, aprender a aprender, interdisciplinariedad del conocimiento y mediación pedagógica. (División de Educación Básica, 2005)

Con el diseño del plan de estudios antes mencionado, el equipo de académicas y académicos de la DEB realiza esfuerzos por trascender los modelos pedagógicos tradicional y conductista, para generar alternativas que consideren los avances científicos y ofrezcan soluciones a los problemas que no han podido ser superados por los modelos mencionados y que, todavía, continúan vigentes en nuestro medio.

La temática de la lectoescritura tiene gran importancia en los planes de estudio de las carreras de esta unidad académica. En la carrera de Pedagogía con énfasis en Educación Preescolar se cuenta con un curso cuyo eje es la alfabetización en el sentido amplio del término, que implica la capacidad de hablar, leer, escribir y pensar en forma crítica y creativa. Este enfoque enfatiza la relación pensamiento-lenguaje, a partir de una concepción de la lectura y la escritura desde su función social. A partir de experiencias de lectura creativa, promueve la recreación literaria, con el propósito de incrementar el interés y el gusto por la lectura en la estudiante maestra, y que ella, con miras a la formación de niñas y niños lectores y escritores autónomos, a su vez favorezca procesos similares con sus estudiantes.

En la carrera de Pedagogía con énfasis en I y II Ciclos, se ofrecen dos cursos para la formación en el área de lectura y escritura. Estos cursos, de igual manera que en la Carrera de Educación Preescolar, ofrecen como eje los procesos de alfabetización y profundizan el conocimiento y análisis de diferentes corrientes innovadoras para la enseñanza y aprendizaje de la lectura y la escritura. Desde ellos, se integran las áreas académicas de la Universidad, por medio de acciones de docencia, investigación, extensión y producción de la lectura y escritura. El estudiantado -maestro o maestra- realiza un proceso de mediación pedagógica con un grupo de tres a cinco niños y niñas con dificultades en lectura y escritura, en una institución educativa pública, desarrollando una serie de propuestas pedagógicas innovadoras. Asimismo, diseña y aplica una propuesta de alfabetización 
para una persona adulta no alfabetizada. Ambas experiencias se sistematizan. Es así como los cursos de lectura y escritura se constituyen en ejes de la práctica, articulando los diversos cursos del nivel, lo cual permite dar coherencia al enfoque establecido en el plan de estudios.

De manera paralela, las profesoras universitarias responsables de esos cursos realizan una serie de talleres de capacitación en lectura y escritura con los docentes y las docentes de aquellas instituciones donde el estudiantado realiza su práctica pedagógica.

\section{Formación docente y aprendizaje con sentido}

El tema de la formación docente en el contexto mundial actual demanda una seria reflexión. La complejidad del papel docente es una realidad porque, efectivamente, el quehacer pedagógico es complejo y de gran impacto social. El docente o la docente de hoy debe estar consciente de la incertidumbre que define nuestro tiempo, y dispuesto o dispuesta a asumir los retos y desafíos ecológicos, de violencia y pobreza, en un mundo caracterizado por la diversidad y el uso creciente de las tecnologías. Para ello debe ser un investigador o una investigadora de su propio contexto educativo y romper con el divorcio entre la teoría y la práctica.

La formación docente debe promover que los maestros y las maestras dejen de pensar que la investigación es sólo para "expertos" y que a ellos y ellas solo les toca "enseñar su disciplina”. Resulta de vital importancia que se posesionen para reflexionar en la acción y sobre la acción pedagógica. Este tipo de formación le permite, a quien ejerce la docencia, dar respuestas pertinentes a las diversas situaciones presentes en la dinámica del aula escolar.

El educador o la educadora, en su formación docente inicial o permanente, debe valorar las interacciones entre su autobiografía personal y el contexto escolar donde labora, entre sus concepciones y las de sus estudiantes, todo lo cual le va a permitir interpretar esa realidad dinámica y promover su enriquecimiento. Por lo anterior, los procesos de formación docente deben estar sumergidos en escenarios reales, en experiencias auténticas que permitan desarrollar miradas críticas y acciones autónomas y creativas.

$\mathrm{El}$ aprendizaje con sentido es un reto para quienes tienen la inquietud de mejorar su práctica educativa en cualquier nivel del sistema. En este caso particular, se hará referencia a la formación docente en el contexto universitario. La propuesta de aprendizaje con sentido se enmarca dentro del constructivismo, lo que implica que el estudiantado es el principal protagonista del proceso pedagógico, con la posibilidad real de construir sus aprendizajes en interacción con los elementos de su entorno. El aprendizaje permite sentir la emoción de descubrir y de entusiasmarse ante lo nuevo. De ahí que se hace indispensable idear estrategias que propicien espacios de construcción, para que las estudiantes y los estudiantes asuman que el aprendizaje les pertenece y, por ende, sientan deseos de aprender.

Se propone, entonces, un docente o una docente con una vasta formación académica y con una alta dosis de sensibilidad para involucrar al estudiantado y conformar entre todas y todos una comunidad aprendiente, donde se construya el conocimiento de manera cooperativa. "La comunidad aprendiente potencia el aprendizaje, permite despertares, desarrolla la capacidad de expresar emociones, recrea ambientes y vive sus tiempos, pues rompe el tiempo y el espacio lineal y posibilita el placer de aprender, la estética, el humor." (Flores, Flores, Jiménez, Madrigal y Perearnau, 2009, p. 41)

La investigadora española Moreno (2003) apunta que “... las emociones también cuentan, los sentimientos, el conocimiento de las otras personas, la capacidad de resolver conflictos y las 
adecuadas relaciones interpersonales", y enfatiza que todo lo mencionado también es inteligencia, pero que no es considerado como tal y, por lo tanto, a esto le llama "subdesarrollo emocional". Agrega que cuando las materias se enseñan desligadas de lo emocional, estas no se aprenden. Por tanto, como alternativa plantea partir de lo cotidiano, no perder la capacidad de sorprendernos, para así romper con la dicotomía mente-emoción.

Moreno (2003) manifiesta que cuando las estudiantes y los estudiantes no saben por qué hacen las cosas, cuando no están motivados o motivadas por lo que hacen y sólo acatan por "obediencia intelectual", se produce una "ceguera cognitiva", con lo cual no hay una participación real del proceso de aprendizaje y, por ende, tampoco se da la construcción de aprendizajes duraderos.

Dentro de este marco de la formación docente, es importante ahondar en la temática de la diversidad presente en el contexto educativo.

\section{Diversidad}

La diversidad ha cobrado especial relevancia en el mundo actual, el cual está cada vez más interrelacionado y en cambio constante; además, esta es parte natural o integral del ser humano. Por lo tanto, se requiere que la pedagogía posesione un enfoque basado en los derechos humanos y en el reconocimiento y respeto por la diversidad, en todas sus dimensiones: de género, socioeconómica, de preferencia sexual, étnica, de procedencia geográfica, de diversas capacidades, o etárea, entre otras.

Lo anterior debe hacerse evidente en las aulas escolares, donde la convergencia de esta diversidad, lejos de ser vista como un problema, se conciba como enriquecedora del desarrollo del currículo, ya que, en ambientes democráticos de aprendizaje, propicia el trabajo cooperativo y solidario.

No se puede negar que el sistema educativo, tradicionalmente, ha privilegiado la homogenización o uniformidad de su quehacer, con la consiguiente exclusión o segregación de quienes no encajan en los criterios establecidos. Esto obedece a la concepción de la realidad que ha prevalecido: una realidad estática, única y con respuestas cerradas, que niega su dinamismo, cambios y constante devenir.

Desde un enfoque basado en el reconocimiento de la diversidad, se habla de igualdad; pero no se trata de una igualdad que uniforme, sino de una igualdad para acceder y disfrutar de los derechos y posibilidades como ciudadanas o ciudadanos. La diversidad implica que todas y todos tenemos diferentes puntos de partida y llegada. Por lo tanto, en el ámbito educativo, esto se debe tener presente para planificar contenidos, propósitos u objetivos, métodos y estrategias que atiendan esta amplia gama de posibilidades, o sea, para implementar un currículo abierto y flexible, donde todas y todos tengan cabida.

En lo referente a la población que se integra en la presente investigación -personas adultas no alfabetizadas- es importante enfatizar en el derecho fundamental que tienen a la educación, independientemente de su edad o discapacidad.

Como lo señala Ruiz (2001), la formación a lo largo de toda la vida -formación permanente o continua- es fundamento de la dignidad de las personas, de su desarrollo personal y socio cultural y de su promoción laboral. Agrega, además, que la educación continua es un instrumento fundamental para combatir la exclusión social y promover una ciudadanía activa, informada y crítica.

Las personas adultas no alfabetizadas y, además, con necesidades educativas especiales o con discapacidad, muchas veces son excluidas de la educación formal y no tienen la oportunidad de 
aprender habilidades básicas para su desenvolvimiento social, tales como la lectura y escritura, lo cual lleva al alejamiento de muchos de sus derechos como seres humanos. Por ello, es importante ofrecerles una nueva oportunidad educativa, que les brinde las herramientas básicas para acceder a la lectura y escritura.

Seguidamente se describe la experiencia desarrollada por las estudiantes y los estudiantes como mediadoras o mediadores de la alfabetización de una persona adulta.

\section{Una experiencia concreta: Escribir juntas y juntos una historia de vida}

Escribir juntas y juntos una historia de vida sintetiza la vivencia de participar en la alfabetización de una persona joven o adulta. "He aprendido a hacer los sueños, realidad", expresa una estudiante universitaria, a lo que agrega una de sus compañeras: "Ahora entiendo que nunca es demasiado tarde para emprender el camino de los sueños". Entrelazar mediación pedagógica y aprendizaje; dejar atrás el determinismo y abrir las puertas a la esperanza, resignificar el papel de la educación en una sociedad que margina por razones de pobreza o de género, son sin duda propósitos fundamentales de esta experiencia.

La alfabetización de personas jóvenes o adultas es un reto que se le presenta al estudiantado que inicia la carrera de Pedagogía, en la División de Educación Básica del CIDE, en la Universidad Nacional de Costa Rica. Es evidentemente un gran reto. Así lo expresan estudiantes una vez concluida la práctica:

- "Este proyecto ha significado un compromiso y un reto por alcanzar, me ha permitido crecer y aprender de los errores".

- "Fue un reto difícil, yo pensaba "es mi primer año, cómo voy a saber" pero uno ve que puede, que muchas veces surge de manera espontánea, que hay que "tirarse al agua".

- "Fue un paso gigantesco para mi formación profesional que de fijo me servirá de mucho. Superé mis temores".

Como se mencionó al inicio de este artículo, en el proyecto que aquí se relata, participaron 68 estudiantes de educación, quienes promovieron la alfabetización de 75 personas. Al invitarles a participar del proyecto como parte de uno de sus cursos, la mayoría manifestó desconocer a personas no alfabetizadas; sin embargo, al poco tiempo estaban realmente sorprendidos y sorprendidas de la facilidad con que las "encontraron"; ello por cuanto muchas de las personas no alfabetizadas ocultan su situación por considerarla como una deficiencia personal, y no como una marginación social.

De las 75 personas jóvenes o adultas participantes, 43 eran mujeres y 32 hombres, sus edades oscilaban entre los 14 y los 90 años, y la gran mayoría (70) eran costarricenses, las 5 personas restantes eran de nacionalidad nicaragüense. En lo referente al estado civil de las personas participantes participaron 53 solteros o solteras, 12 casados o casadas, 4 viudos o viudas, 3 en unión libre, y 2 divorciados o divorciadas. La ubicación geográfica tiene que ver con la provincia donde viven las estudiantes o los estudiantes universitarios. Así se obtuvieron 24 en Alajuela, 22 en Heredia, 17 en San José, 6 en Cartago, 3 en Limón, 2 en Guanacaste, y 1 en Puntarenas. Cabe destacar que las estudiantes o los estudiantes que vivían lejos de la universidad, realizaban la alfabetización durante los fines de semana, cuando asistían a su comunidad. Es interesante subrayar que 13 de las personas participantes eran familiares de las estudiantes o los estudiantes universitarios, 12 eran vecinos o 
amigos, y 9 eran solamente "conocidos"; de ello se desprende, entonces, que 41 participantes no tenían ninguna relación previa con el estudiantado de la universidad.

Un dato importante en un proceso de alfabetización es la escolaridad de sus participantes. En el caso descrito, se encontró que 30 personas no asistieron nunca a la escuela, 20 asistieron a instituciones de educación especial debido a su discapacidad, y el resto asistió a la escuela; de ellos y ellas 10 únicamente estuvieron en primer grado, 7 en segundo grado, 3 en tercer grado, 1 en cuarto grado, y 4 llegaron hasta sexto grado. Respecto a su ocupación, 26 personas asistían a un Taller protegido, lugar al que concurren diariamente personas adultas con alguna discapacidad; 14 eran amas de casa, 12 se encontraban desocupados, 6 retirados, 5 realizaban labores como empleadas domésticas, una tenía trabajos ocasionales, otro era un comerciante y 10 personas se dedicaban a diversas labores.

Durante el primer semestre del año lectivo, las estudiantes y los estudiantes universitarios estudian diferentes corrientes innovadoras para el aprendizaje de la lectura y la escritura, así como los procesos de conceptualización de la escritura, lo que les permite profundizar en la utilización de alternativas metodológicas y generar sus propias propuestas de mediación pedagógica. En ese ciclo realizan un proceso de intervención pedagógica, el cual sistematizan, con un grupo de tres a cinco niños y niñas con dificultades en lectura y escritura, en una institución educativa pública. Se busca que el estudiantado comprenda los procesos de construcción de la lectura y escritura, y promueva su aprendizaje en contextos funcionales y significativos, para lo cual se abordan los siguientes temas:

- El proceso de alfabetización inicial, en el contexto familiar, comunitario, social y cultural de niños, niñas y personas jóvenes y adultas no alfabetizadas.

- Función social y emancipadora de la lectura y escritura.

- El aprendizaje de la lectura y escritura como proceso evolutivo: Los niveles de conceptualización de la escritura y su integración en la mediación pedagógica.

- Corrientes pedagógicas innovadoras para la atención de los procesos iniciales de lectura y escritura.

- Alfabetización de personas adultas.

- Estrategias lúdicas para promover el aprendizaje de la lectura y escritura.

Asimismo, en ese ciclo lectivo diseñan una propuesta de alfabetización para una persona joven o adulta, para lo cual requieren establecer los primeros vínculos con quien realizarán la experiencia. Cada estudiante inicia el proceso en el momento en el cual se considere preparado o preparada, lo que se ve influido por el interés y "urgencia" que le exprese la persona con quien trabajará. El diseño del proyecto es un documento abierto, con pautas generales de acuerdo con las características de cada persona. Empiezan conociendo las razones por las cuales esta persona no ha aprendido a leer y escribir, y las motivaciones para emprender nuevamente la aventura.

Las razones a las que atribuyen no haber aprendido quienes participaron en este proceso son en 28 de los casos de índole económica, 27 aluden a su discapacidad, 6 a problemas familiares, 5 a discriminación por género dado que por ser mujeres no les permitieron estudiar, 5 mencionan argumentos referidos a la metodología que utilizaba su maestra, 2 a la distancia de su casa a la escuela, y 2 a desmotivación personal.

En cuanto a la motivación para aprender, 44 personas indican que es para lograr su superación personal, 6 tienen como objetivo leer la Biblia, mientras que 4 personas quieren leer el periódico y revistas, 5 desean aprender a leer y escribir para conseguir trabajo, 2 para ingresar al INA, 1 para obtener la nacionalidad costarricense, 4 para mejorar la comunicación, 2 para tomar el bus, 
una para utilizar el teléfono celular y otra persona desea leer la información que se ofrece en el supermercado.

A manera de ilustración, de los datos indicados anteriormente, transcribimos de seguido algunas expresiones de estudiantes universitarias o universitarios:

- "Ella lo que desea es aprender para hacer mejor los mandados y ahora que su esposo está enfermo aprender para poder leer las instrucciones del hospital y poder cuidarlo bien".

- "Quiere leer los mensajes en el cuaderno de recados de su hijo".

- "Tiene como meta estudiar en el INA".

- "Está interesada en enseñarle a sus sobrinos."

- "Quiere obtener la nacionalización costarricense y para ello quiere aprender para hacer dos exámenes que le solicitan".

En la formación del estudiantado, se enfatiza la importancia de partir de los intereses y necesidades, así como de los conocimientos previos de quien aprende, y respetar los tiempos de aprendizaje de cada persona. Por ello, es vital llevar a cabo un diagnóstico para conocer las producciones que son capaces de realizar quienes participan en un proceso de alfabetización inicial, así como el nivel de conceptualización de la escritura que poseen en ese momento.

Es necesario tener presente que, como se indicó anteriormente, cincuenta y una de las personas participantes presentan algún tipo de necesidad educativa especial. Así tenemos que al inicio del proceso de alfabetización 8 personas solamente eran capaces de elaborar garabatos, 22 sólo escribían algunas letras o sílabas aisladas, 9 únicamente su nombre, 11 su nombre y apellido, 18 su nombre completo y algunas palabras, 6 textos cortos, y solamente una persona podía leer y escribir, aunque con dificultad, textos amplios.

En cuanto al nivel de conceptualización de la escritura, 10 personas se encontraban en el nivel concreto, o sea no podían distinguir el dibujo de la escritura; 25 se hallaban en el nivel simbólico, -ya diferenciaban lo que eran dibujos de las palabras, pero no habían descubierto la relación entre el texto y los aspectos sonoros del habla-; 27 se encontraban en el nivel silábico y, por lo tanto, escribían una letra o pseudo-letra por cada sílaba emitida; 8 personas estaban en el nivel alfabético inicial, así que podían establecer una correspondencia entre los fonemas que forman una palabra y las letras necesarias para escribirla, pero entendiendo la sílaba únicamente formada por una consonante y una vocal; y finalmente 5 personas estaban en el nivel alfabético medio, comprendiendo las sílabas formadas por consonante, vocal, consonante, o por consonante, consonante, vocal.

De esta experiencia vivida por el grupo de estudiantes, emergen valiosos aprendizajes para su formación docente, los cuales se describen a continuación.

\section{Aprendizaje en la docencia}

La práctica de alfabetización de personas jóvenes y adultas, que realizan las estudiantes y los estudiantes universitarios, tiene entre sus propósitos generar espacios para la construcción de aprendizajes que impacten la formación de docentes críticos e innovadores. Una vez concluido el proyecto, se realizó una indagación a partir de la pregunta: ¿Qué ha aprendido usted en la experiencia de alfabetización de la persona adulta? De las respuestas obtenidas emergen las categorías que se exponen seguidamente, las cuales se ilustran con expresiones del estudiantado: 
1. Valorar las oportunidades, superar los obstáculos

"Aprendí que a pesar de que uno tiene alguna discapacidad, no tiene por qué dejar que sus objetivos no se cumplan, que cada esfuerzo que se hace vale la pena".

2. La edad no es impedimento para aprender

"Aprendi que no importa la edad que uno tenga, si uno tiene ganas de superarse y aprender, se puede lograr".

3. Desarrollar paciencia, perseverancia, humanismo, sensibilidad, solidaridad, creatividad, fe, optimismo, tolerancia, valorar las diferencias

"He aprendido el valor de la paciencia, de la perseverancia, el valor de la amistad".

4. Considerar los intereses, necesidades y contexto

"He aprendido que una persona aprende no de acuerdo a como yo creo que aprende, sino según su contexto, su forma de vida, sus actitudes, aptitudes y capacidades".

5. Que al enseñar también se aprende

"Me pude dar cuenta que al igual que yo le enseñé a ella muchas cosas que le servirán para toda su vida, ella también me enseñó a mí a ver la vida desde otro punto de vista".

6. La importancia que tiene el ser alfabetizado

"Aprendi que leer y escribir es un derecho que debe ser universal".

7. A escuchar, a compartir, valorar la experiencia del aprendiz

"He aprendido a escuchar y a relacionar las experiencias de esta persona con mi vida".

8. Valorar la carrera de educación, sentirse útil, influir en la vida de las personas

"Aprendí que estoy en la carrera indicada, que es esto lo que yo quiero para mi vida, y a sentirme útil".

En las reflexiones que se realizaron en el aula universitaria se enfatizó la importancia de respetar las características de las personas participantes en un proceso de alfabetización, por lo que se sistematizaron las respuestas del alumnado a las preguntas:

¿Cómo ha respetado usted la diversidad de la persona adulta que alfabetiza?

¿Qué estrategias ha utilizado en atención a las características particulares de esta persona?

De ellas surge la siguiente información:

1. Partiendo de necesidades e intereses de las personas adultas

"He utilizado cosas propias de esa persona: como es cocinera de escuela le gusta mucho cocinar, le enseño recetas o el menú de la semana".

2. Utilizando juegos

"Usamos muchos juegos, como bingo, juegos de armar y desarmar". 


\section{Siendo paciente}

"Creo que la estrategia más importante ha sido llenarme de paciencia, trabajamos en forma tranquila sin apuros, le hablaba despacio y vocalizaba mejor las palabras".

\section{Recurriendo a la conversación, al diálogo}

"Tuve que usar mucho la conversación".

\section{Manejando diversos métodos}

"Empecé con el nombre propio, luego con el nombre de sus familiares".

\section{No tratándole como niño o niña}

"No deseaba sentirse como un niño, quería ser tratada como una persona adulta".

\section{Atendiendo sus necesidades de horario.}

"Fue necesario flexibilidad en el horario".

Aprender a leer y escribir es un proceso liberador del ser humano, y ello se profundizó invitando al estudiantado a seleccionar y leer un libro de Paulo Freire, el que cada estudiante quisiera, y luego cada uno o una compartió sus reflexiones de manera creativa. Así, algunos elaboraron una poesía, otros presentaron un monólogo, dos estudiantes se expresaron por medio de la danza, otros con dibujos, fotografías o caricaturas; en fin, cada uno o cada una haciendo valer su libertad de expresión. Posteriormente se les preguntó ¿En qué sentido ha sido liberador para la persona adulta que usted alfabetiza, el aprender a leer y escribir? De cuyas respuestas obtuvimos:

\section{Realizar actividades cotidianas de manera autónoma}

- "Ya puede ir al supermercado y comprar algo sin preguntar qué dice en el envase."

- "Ha sido liberador a la hora de ir a la calle y tomar un bus y leer para dónde va".

- "Ahora puede leer los rótulos de la calle".

- "Ya no le da vergüenza hacer las compras".

- "Ella tiene una libreta con nombres y números de teléfono, siempre me decía que le buscara alguno, y ahora ella puede sola”.

\section{Comunicarse o expresarse mejor}

- "Tanto él como yo estamos como liberándonos".

- "Ella se siente libre de poder escribir lo que quiera".

- "Puede escribir las colillas de regalos".

- "Ha aprendido mucho y no es solo la satisfacción de saber leer y escribir sino el "poder", el decir "yo puedo hacer las cosas".

\section{Sentirse valorado, feliz y realizado, integrado en la sociedad}

- "Ella expresa mucha alegría al poder escribir su nombre".

- "Está muy feliz de haber cumplido la meta a pesar de que tenía mucho miedo de fracasar".

- "Ya no se sentía como un estorbo en la sociedad, sino que ya podía desenvolverse como todas las demás personas de su entorno".

- "Algo que ha sido muy significativo para él es que su familia se siente muy orgullosa de él". 
- "Ahora puede leer la Biblia junto con los de su Iglesia".

- "Le ayudó a sonreír cada vez que aprende algo nuevo".

- "Cuando hay algún rezo se anima a leer alguna oración”.

\section{Le abre las puertas a nuevos aprendizajes o actividades}

- "Lo ha impulsado a estudiar lo que a él le apasiona que es la mecánica automotriz".

- "Ella tiene un gran interés por estudiar y a aprender... y el saber que ella puede fue una de las cosas más importantes que aprendió”.

\section{Desarrolló confianza en sí mismo o sí misma}

- "Ya puede escribir los nombres de sus hijos".

- "Ahora puede salir con confianza a la calle".

- "Puede sentir más confianza al atender a un proveedor y hacer la lista de compras ella misma."

- "Ahora es una persona más independiente, ya que antes no salía sola porque sentía temor".

- "Le aumentó la autoestima y logró darse a respetar"

\section{Le permitió leer lo que quiera}

- "A leer un libro de recetas que ella quería leer y lo tenía guardado".

- "Siempre que quería leer un cuento se lo pedía a algún familiar".

- "Le motiva leer y compartir cuentos con sus hijos."

También nos interesó conocer cuáles fueron los momentos más significativos para las estudiantes y los estudiantes universitarios, en la experiencia de alfabetización llevada a cabo, y fue así como obtuvimos las siguientes respuestas:

\section{Relacionadas con aprender a leer y escribir}

- "Cuando escribió su nombre sola".

- "Cuando Lency empezó a escribir las pequeñas oraciones que yo le dictaba sin muchos errores".

- "Al verlo escribir y reconocer su nombre".

- "Cuando realizamos las lecturas y leímos unas cuantas recetas".

- "Al verle escribir el nombre y apellidos de sus hijos y que logró regalarle un cuento a la nieta".

- "El saber que esta persona avanzó y ya puede leer textos cortos y sencillos”.

- "Cuando en el bus iba leyendo letreros, me contó que quería leer la Biblia y me mandó una carta dándome las gracias".

- "Cuando le enseñé las vocales y las pudo diferenciar y escribir".

- "Cuando escribió el nombre de sus tres hijos y fuimos al supermercado y ella leía los nombres de lo que íbamos a comprar ".

- "Cuando me dijo que sí quería aprender el nombre y se le pusieron los ojos llorosos".

- "Cuando logró escribir sus propias caricaturas".

- “Cuando leyó por fin”.

- "Cuando Aarón comenzó a leer con más fluidez”.

- "Cuando leyó sus primera palabras".

- "Cuando logró hacer una historia amplia y leer todo un cuento". 


\section{Relacionadas con deseos de aprender y entusiasmo}

- "Por el entusiasmo que siempre mantuvo y las ganas de aprender".

- "Donde me di cuenta de las ganas enormes de aprender".

- "Saber que doña María le había pedido a Dios que le pusiera en su camino a alguien que la pudiera guiar en la lectura y escritura”.

- "Compartir con ella la ilusión que tenía de subirse a un avión”.

- "Las ganas que le pone para aprender".

- "Ver el esfuerzo y perseverancia que Jairo demostró siempre”.

\section{Relacionadas con un cambio en la vida de la persona adulta}

- "Ahora noto un cambio de actitud que ella tenía hacia ella misma".

- "Cuando pudo leer una carta que le habían dado hace mucho tiempo".

- "Le ha ayudado muchos con los buses y ya no se siente mal, porque antes le preguntaba al chofer y él le decía "ahí dice, qué es que no sabe leer".

- "Ya no lo molestan como antes y tuvo el apoyo de su novia, familia y amigos".

- "Cuando expresa que ahora si va a poder hacer muchas cosas".

- "Cuando me cuenta que sus hijos le dicen: "mami qué lindo escribe".

- "Cuando me ha contado que puede leer el obituario en el TV”,

- "Jairo es más extrovertido y ahora habla más”.

4. Relacionadas con aprendizajes para el estudiante o la estudiante que alfabetizó

- "Cuando conocí a la persona ya que la interacción me llenó de mucha esperanza por querer hacer algo por otra persona".

- "Ella me abrazó y me dijo que me agradecía mucho la ayuda, me hizo sentir muy bien... me siento orgullosa de que haya sido mi primera alumna".

- "Entendi que la alfabetización es muy importante y por eso como futura docente espero lograr que los niños aprendan bien”.

- "Las palabras que me ha dicho y el apoyo que me ha dado, la mirada de agradecimiento cada vez que puede leer una palabra".

- "Testimonios que son fuertes y motivadores para la vida de uno, como otros han podido surgir a pesar de las adversidades”.

- "La gratificación de estar ayudando a mi hermano a superarse como persona”.

- "El reto de enseñarle a una persona de la misma edad de mi mamá".

- "Aprendí que las personas con discapacidad son personas muy especiales, capaces e inteligentes".

- "Conocer su cultura (Nicaragua)".

- "Hubo momentos en que me inspiraba el esfuerzo e interés que ella mostraba".

- "El día que su mamá me visitó para manifestarme su agradecimiento".

- "Me ayudaron para mi vida personal y mi formación docente”.

\section{Todo el proceso fue significativo}

- "Todo el proceso ha sido para mi muy significativo".

- "Todos los momentos han sido muy importantes".

- "En cada sesión hubo momentos significativos".

- "Es un proceso que deseo continuar".

- "Toda la experiencia significó mucho para mi”. 
Para concluir, preguntamos directamente a cada estudiante ¿Qué ha significado para usted esta experiencia de alfabetización en su proceso de formación como educadora o educador? Sus respuestas aluden a la importancia de enfrentar nuevos retos, a la sensibilización y valoración de las diferencias que desarrollaron, así como al acercamiento a la realidad, lo cual les permitió aprender cómo ser docentes y disfrutar la carrera de educación. También hacen referencia al valor de esa experiencia para su vida personal y agradecen a Dios por la oportunidad del aprendizaje vivido. En sus propias palabras, ello se expresa de la siguiente manera:

- "Quiero comentar que esta ha sido una de las experiencias más significativas y que ha marcado mi vida".

- "Me ha servido de mucho. Es una de las experiencias más enriquecedoras de mi vida. Me ha hecho asegurarme de que estoy en la carrera adecuada."

- "Grandiosa, tengo más confianza en mí y en mi capacidad."

- "Una experiencia vital, me acercó a la realidad”.

- "Me ha servido para valorar muchas cosas, entre ellas el esfuerzo de mis padres de mandarme a la escuela".

- "La experiencia me enseñó, que como futuro educador tengo un compromiso con todas aquellas personas que no están alfabetizadas. Además descubrí que mi labor como educador va más allá que las lecciones que dé dentro de una escuela”.

- "Aprendi que se debe dejar que la lección sea lo más natural posible."

- "Significó una alegría para mí y un orgullo de que a pesar de ser mi primer año en la U, ya pueda enseñar a leer y a escribir a alguien”.

- "Ahora comprendo mucho mejor el gran significado que representa el enseñarle a los niños(as) a aprender a leer y escribir".

- "Aprendí a considerar la importancia de enseñar con respeto y solidaridad."

- "Tenemos que educar de acuerdo con los intereses y capacidades de las demás personas, también me ha puesto a reflexionar que no todos los seres humanos tenemos que aprender de la misma forma."

- "Fue muy significativo porque aprendí a relacionarme con un adulto no alfabetizado que ha ideado formas para adaptarse al medio sin saber leer, aprendi a utilizar materiales auténticos".

- "Fue una experiencia enriquecedora, aprendí que alfabetizar es una experiencia grandiosa y maravillosa".

- "Tuve la oportunidad de trabajar con mi abuela materna, ella me cuidó y alimentó, ahora yo hice algo de mucho valor para ella”.

- "Este proyecto nos ayuda a tener otras expectativas, a abrir los ojos a la realidad en las que se encuentran nuestras escuelas y nuestros alumnos".

- "Definitivamente todos los futuros docentes deben tener una experiencia de este tipo para reafirmar su vocación y tener nuevas formas de pensar".

- "Alfabetizar implica dar y recibir".

- "Este proceso nos ayuda a sensibilizarnos y crear una mejor sociedad".

- "Me ha afectado profundamente ver cómo la persona analfabeta se desvaloriza a sí misma, ahora sé que toda persona merece ser alfabetizada, devolverle la esperanza." 


\section{Reflexión conclusiva}

Hemos podido constatar que las estudiantes y los estudiantes, al realizar una experiencia de alfabetización con una persona adulta con las características descritas, comprenden el compromiso que conlleva la educación y se entregan a la docencia con pasión, dejando atrás miedos y prejuicios, para crear espacios participativos de construcción de conocimientos.

La ejecución de una experiencia pedagógica en una situación real y con una persona joven o adulta deseosa de aprender a leer y escribir, ofreció al grupo estudiantil la oportunidad de valorar las oportunidades que ellas y ellos han tenido para acceder al nivel universitario, así como reflexionar respecto a los condicionamientos sociales, económicos y culturales que excluyen de la educación a diversos sectores de la población. Esa experiencia les permitió también visualizarse como futuras y futuros profesionales que requieren buscar alternativas innovadoras para propiciar aprendizajes significativos en sus estudiantes y, sobre todo, descubrirse como seres humanos que pueden compartir de manera solidaria con otros seres humanos.

Como educadoras que apoyamos la experiencia de alfabetización de personas adultas descrita, $\mathrm{y}$ que mediamos el proceso formativo de futuras y futuros docentes, podemos decir que con esta práctica reafirmamos nuestra creencia en la posibilidad de que la educación abra nuevas esperanzas hacia formas de relación social más equitativas; nuevas oportunidades y nuevas miradas, para la edificación de sociedades más solidarias, más democráticas y más creativas.

\section{Referencias bibliográficas}

División de Educación Básica. (2005). Plan de Estudios de la Carrera de I y II Ciclos. Costa Rica: Universidad Nacional.

Flores, L. E., Flores, G., Jiménez, R. E., Madrigal, J. C. y Perearnau, M. (2009). Comunidad Aprendiente. Costa Rica: ARTEMUSA.

Moreno, M. (2003). Las ausencias de la enseñanza. Conferencia, Universidad Estatal a Distancia, Costa Rica.

Ruiz, A. (2001, Enero). Materiales previos. [Grupo de trabajo]. Realizado en el Congreso 2001: Construir la escuela desde la diversidad y para la igualdad. Madrid: España. Disponible en http://www.nodo50.org/igualdadydiversidad/g_contin.htm

Universidad Nacional. (1993). Estatuto Orgánico. Costa Rica: UNA-Gaceta. 